\title{
Strategies and Mechanisms in Nonselective and Selective Inhibitory Motor Control
}

\author{
Ritske De Jong \\ University of Groningen
}

\author{
Michael G. H. Coles and Gordon D. Logan \\ University of Illinois at Urbana-Champaign
}

\begin{abstract}
Motor inhibition was studied in 3 versions of the stop-signal paradigm, with the stop signal requiring inhibition of any response (stop-all), a fixed alternative response (stop-change), or selective inhibition of only 1 of the responses (selective-stop). The lateralized readiness potential was used in Experiment 1 to distinguish between a selective, central, and a global peripheral inhibition mechanism. Inhibition was found to be effected by the central mechanism in the stop-change condition and by the peripheral mechanism in the other conditions. Manipulation of stimulus discriminability in Experiment 2 strongly affected the speed of selective motor inhibition, confirming that such inhibition was achieved by conditionally engaging the peripheral mechanism. These results support the idea that functionally distinct mechanisms and strategies are involved in inhibitory motor control in different situations.
\end{abstract}

People are able to withhold, interrupt, or rapidly change planned or ongoing actions when these actions are suddenly rendered inappropriate by unanticipated changes or events. This ability has great functional significance and is also of considerable theoretical interest because it involves an internally generated inhibitory intervention or act of control by which overt movement is temporarily halted or redirected. The study of inhibitory motor control may thus provide an important source of information about the nature of executive processes that dynamically regulate the operations of the human information-processing system. The role of inhibition in motor control is also interesting in its own right. The cerebellum and basal ganglia-midbrain structures that play an important role in motor controlboth exert their influence by inhibitory modulations (Brooks, 1986). Adaptive movements thus appear to depend on an intricate and, at present, poorly understood interplay between excitatory and inhibitory mechanisms by which certain movements or muscle synergists are activated and others actively inhibited. Motor activation and inhibition processes will be shown to operate largely independently in the tasks used in the work reported here. This fact makes it possible to examine the individual functional characteristics of these processes and provides a useful starting point for

Ritske De Jong, Department of Experimental and Occupational Psychology, University of Groningen, Groningen, The Netherlands; Michael G. H. Coles and Gordon D. Logan, Department of Psychology, University of Illinois at Urbana-Champaign.

The research reported in this article was supported by Grant MH41445 from the National Institute of Mental Health.

Correspondence concerning this article should be addressed to Ritske De Jong, Department of Experimental and Occupational Psychology, University of Groningen, Grote Kruisstraat 2/1, 9712 TS Groningen, The Netherlands. Electronic mail may be sent via Internet to r.de.jong@ppsw.rug.nl. the study of more complex modes of interaction between these processes in the performance of less constrained motor tasks.

Motor inhibition has been investigated with the stopsignal paradigm, in which participants perform a speeded reaction task and are occasionally presented with a stop signal that instructs them to withhold the response (for a review, see Logan, 1994). Performance in the stop-signal paradigm can be modeled in terms of a race between two sets of processes that operate independently; one set starts with the onset of the task stimulus and results in the activation and execution of the response, whereas the other set starts with the onset of the stop signal and results in the inhibition of the response (Logan \& Cowan, 1984; Osman, Kornblum, \& Meyer, 1986). The response is made or withheld, depending on which set of processes wins the race. Consistent with the race model, participants are increasingly better able to inhibit the response as the delay between the task stimulus and the stop signal (stop-signal delay) is shortened.

We used continuous-response measures in a previous study to show that speeded manual responses could be inhibited rapidly and at any time during the course of processing in a speeded-reaction task (De Jong, Coles, Logan, \& Gratton, 1990). An important question in that study concerned the way in which rapid motor inhibition was effected. Event-related brain potentials were used to examine inhibitory effects on response-related activity patterns in cortical motor structures. Though activity in these structures was found to be subject to inhibition, demonstrating the operation of a centrally operating inhibition mechanism, two additional findings suggested that invocation of this mechanism was not the primary means by which inhibition of the overt response was accomplished. First, the earliest signs of central motor inhibition occurred only after inhibition of the overt response was already completed 
or well underway, as indicated by the electromyogram (EMG) and continuous-response measures. Second, even when the overt response was completely inhibited, activity levels in cortical motor structures frequently exceeded those normally associated with the onset of overt movement.

On the basis of these findings, we hypothesized that two mechanisms are available to interrupt or withhold speeded responses, a central (cortical) mechanism and a more peripherally operating mechanism. The central mechanism operates by inhibiting response-activation processes in cortical motor structures in order to prevent central outflow of motor commands. The peripheral mechanism operates by preventing the actual execution of central motor commands by peripheral motor structures, possibly by blocking the transmission of such commands. The idea of a peripherally operating inhibition mechanism has received support from recent results by Jennings, van der Molen, Brock, and Somsen (1992). They found that the interval between heart beats was prolonged when the motor response was successfully inhibited as compared with when inhibition failed. The fact that cardiac inhibition and motor inhibition interact is consistent with the notion that they were controlled in part by the same midbrain system.

The distinction between a central and a peripheral inhibition mechanism is consistent with that between central and peripheral motor processes proposed by Bullock and Grossberg $(1988,1991)$ in their model for the control of limb movements. These investigators suggested that central processes are concerned with the programming of structural aspects of movements, such as their direction and amplitude. More peripherally operating processes generate a "GO" signal that interacts with the central motor commands in a multiplicative fashion in order to produce the actual commands sent out to the motorneuron pool. Peripheral processes are thus concerned with the energetical aspects of the movement, controlling its onset and speed. Rapid motor inhibition can then be achieved by inhibiting the GO signal (Bullock \& Grossberg, 1988).

The idea that a peripherally operating mechanism may yet serve to withhold movements, even when central motor outflow cannot be prevented, is appealing but must be regarded as speculative because the empirical evidence for it is still scant. Moreover, the interpretation of much of this evidence rests on a number of assumptions about the functional relationships between physiological mechanisms and measures and psychological processes; the validity of these assumptions often remains somewhat uncertain (Logan, 1994; Meyer, Osman, Irwin, \& Yantis, 1988; van der Molen, Bashore, Halliday, \& Callaway, 1991). The major aim of the present research was to put this idea to a direct test and to assess the functions and dynamics of the two inhibition mechanisms in some detail.

\section{The Present Research}

The peripherally generated GO signal in Bullock and Grossberg's (1988) model is assumed to operate in a largely nonspecific way, shunting or multiplying any commands issued by central motor processes. A nonspecific mode of operation ensures smooth and straight trajectories for movements involving several different muscle groups and contributes to the temporal coordination of actions that involve different effector systems (Bullock \& Grossberg, 1988). However, it would also seem to limit the use of the peripheral inhibition mechanism, which supposedly inhibits the GO signal, to situations where inhibition is all-or-none, resulting in behavioral arrest. Such situations are not uncommon. For instance, complete behavioral arrest is characteristic of startle and orienting responses that occur in reaction to unexpected and perceptually salient events (Rohrbaugh, 1984). The basic stop-signal paradigm, in which the stop signal indicates the need to stop any motor response, is representative of situations of this type. There are many other situations, however, that require more deliberate inhibitory control by which some undesired responses are inhibited, whereas others proceed unimpeded. According to the present hypothesis, the central inhibition mechanism should subserve inhibition in such cases, as only this mechanism is presumably capable of selectively preventing the flow of motor commands from central to peripheral motor structures, by selectively inhibiting the issuing of central commands for undesired responses while leaving the GO signal in effect so that desired responses may be executed.

These considerations motivated the present research, which had two related goals. The first goal was to extend the study of motor inhibition from all-or-none situations, as in the basic stop-signal paradigm, to those in which selective motor inhibition is required, with some responses being inhibited but not others. The second goal was to put the notion of two functionally distinct inhibition mechanisms to a rigorous test. We tested two predictions derived from the two-mechanism model of motor inhibition by comparing the dynamics of motor inhibition in situations where it can be all-or-none with those in situations where it needs to be selective.

The first prediction was based on the assumption that peripheral inhibition is faster than central inhibition (De Jong et al., 1990) and held that, everything else being equal, attempts to inhibit are more likely to be successful if the peripheral mechanism can be used. This likelihood is because even when the stop signal occurs too late for the central mechanism to be able to prevent central motor outflow, the peripheral mechanism may still be able to prevent the overt response by blocking this outflow "downstream." The second prediction focused on the different implications of the two mechanisms regarding the relation between activity levels in central motor structures and the presence or absence of overt movement. When inhibition is accomplished by means of the central mechanism and the peripheral GO signal remains in effect to energize any ensuing central motor command, it is crucial that activation levels in cortical motor structures do not exceed those at which outflow of central commands occurs. Consequently, if the response is successfully inhibited, central response activation should remain well below levels normally associated with the initiation of overt movement. In contrast, 
when motor inhibition is effected by the peripheral mechanism, by inhibiting the GO signal, central activation levels on signal-inhibit trials should be able to exceed those normally associated with response initiation, as was indeed observed by De Jong et al. (1990).

\section{Race-Model Analyses}

In the experiments reported here, speed of inhibition was assessed by estimating the stop-signal reaction time (SSRT), which represents the time for the internal inhibitory response to the stop signal to become effective. The estimation procedure was based on the horse-race model for the stop-signal task and has been described in detail by Logan and Cowan (1984); because the procedure is of central importance in the present work, a brief description will be provided here. Two assumptions are made in the race-model analyses. First, primary-task processing and stop-signal processing are assumed to operate independently. This assumption actually entails two separate assumptions: (a) the primary-task reaction time (RT) distribution is the same whether the stop signal is present or absent (signal independence), and (b) when a stop signal is present, the finishing times for primary-task processing and stop-signal processing are uncorrelated (stochastic independence). Second, the SSRT is assumed to be constant. Though the latter assumption is unlikely to be valid in a strict sense, Monte Carlo simulation results by De Jong et al. (1990) have indicated that violations have only minor effects on the accuracy of the predictions and estimates derived from the race model.

Under these assumptions, the RT distribution for trials on which no stop signal occurred (no-signal trials), in conjunction with the probability of responding given a stop signal, can be used to predict mean RT for trials on which a stop signal occurred but the participant failed to stop (signalrespond trials) and to estimate SSRT. As depicted in Figure 1 , the effect of the stop signal can be thought of as dividing the no-signal RT distribution into two parts. The left part corresponds to the probability of responding given a stop signal and consists of responses fast enough to escape inhibition; the right part corresponds to the probability of successful inhibition. Mean RT for signal-respond trials should then exactly match the mean of the left part of the no-signal RT distribution. The cutoff point represents the point in time at which the internal response to the stop signal is thought to occur, relative to the onset of the primary-task stimulus; subtracting out the stop-signal delay then yields an estimate of SSRT relative to the onset of the stop signal.

\section{Derivation of the Lateralized Readiness Potential}

Following the same approach as used by De Jong et al. (1990), the lateralized readiness potential (LRP) was used as a measure of central motor activation. This measure is based on the fact that unimanual responses are associated with greater activity in the precentral motor cortex of the hemisphere contralateral to the response hand and consequently with a larger negative potential recorded from the scalp directly above that part of the motor cortex. The difference between the scalp potentials recorded above the hand areas in the left and right motor cortices then provides a measure of the relative central activation of the two response hands. However, this measure may be contaminated by nonmotor functional differences between the two hemispheres. Such contaminations can in most cases be effectively removed by subtracting the averaged potential difference for left-hand responses from that for right-hand responses, resulting in the LRP. This subtraction removes hemispheric differences of nonmotor origin, as these can generally be assumed to be the same for left- and right-hand responses, while preserving those differences that are specifically related to the central activation of the manual responses. (For a detailed explanation of the derivation of the LRP and its use in mental chronometry, see Coles, 1989.)

\section{Experiment 1}

In this experiment, we used three different versions of the stop-signal paradigm. These versions and the relevant predictions for each will be discussed in turn.

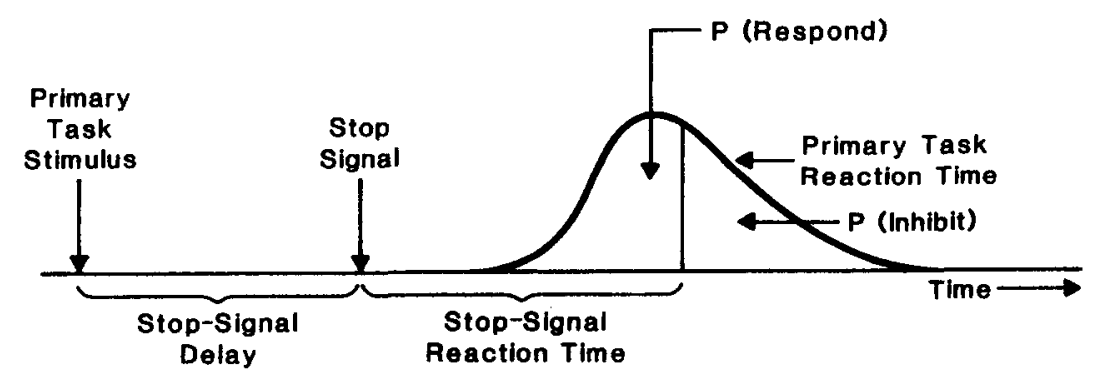

Figure 1. Illustration of the race model for response inhibition. Depicted is a hypothetical no-signal reaction time distribution. The distribution is divided into two parts, with the left part corresponding to signal-respond and the right part to signal-inhibit trials. The location of the cutoff point in the distribution depends on the stop-signal delay and the stop-signal reaction time. $\mathrm{P}=$ probability. 


\section{Stop-All Condition}

In this condition, we used the standard procedure in which the stop signal summons the inhibition of any response. Previous results (De Jong et al., 1990; Jennings et al., 1992) suggest that motor inhibition in this condition is primarily accomplished by means of the fast peripheral mechanism. Thus, in this condition we expected to replicate our previous finding of a partial functional dissociation between activity levels in central motor structures, as measured by the LRP, and overt movement, in the sense that overt movement can be completely absent even when the LRP reaches or exceeds amplitudes that are normally (in the absence of the stop signal) associated with overt movement.

\section{Stop-Change Condition}

In this condition, the stop signal required participants not only to inhibit the response to the task stimulus but also to execute immediately a fixed alternative response. This variant of the stop-signal paradigm was used by Logan and Burkell (1986; see also Logan, 1985) to assess the role of response competition in dual-task interference. We used it here as a way to delimit the usefulness of the peripheral inhibition mechanism. Because inhibition of the GO signal by the peripheral mechanism would temporarily prevent all motor outflow, the requirement to make an immediate alternative response to the stop signal itself was hypothesized to necessitate the use of the central mechanism to selectively inhibit the primary-task response.

SSRTs were predicted to be longer in the stop-change than in the stop-all condition. Longer SSRTs in stopchange conditions were in fact found by Logan and an associate (Logan, 1985; Logan \& Burkell, 1986). However, this difference might reasonably be attributed to the requirement to make two responses to the stop signal-an internal inhibitory response and an overt motor response-and, taken by itself, does not compel the conclusion that different mechanisms were used in the two conditions. It is here that the LRP measure becomes particularly relevant. If, as we predicted, the central inhibition mechanism is used in the stop-change condition, then LRP amplitudes on trials where the response is successfully inhibited (signal-inhibit trials) should remain well below levels associated with initiation of overt movement and be considerably smaller than the LRP amplitudes observed on signal-inhibit trials in the stop-all condition. Confirmation of this prediction would lend strong support to the two-mechanism hypothesis.

The fixed motor response to the stop signal involved either pushing or releasing foot pedals with both feet simultaneously. This choice was motivated by the requirement that this response not interfere with the measurement of the LRP associated with the manual primary-task response. Given that the LRP reflects a difference between left-hand and right-hand responses, the fact that the same, fixed foot response was always required goes far toward meeting that requirement. (See Osman \& Moore, 1993, for a similar use of hand-foot response combinations in LRP measurements.) The simultaneous use of both feet was intended to eliminate possible body side-specific interactions between the manual and foot responses. As the results will demonstrate, this procedure appeared to work very well.

\section{Selective-Stop Condition}

In this condition, the stop signal required participants to inhibit responses made with one hand, referred to as the critical hand, but not those made with the other, noncritical hand. The central inhibition mechanism would seem to be well suited for this purpose. In an earlier study that used this version of the stop-signal paradigm, Logan, Kantowitz, and Riegler (1986) found that participants tended to selectively delay the critical response in order to enhance their chances of being able to inhibit this response when required. This finding demonstrates that people can adopt and maintain a tonic selective inhibitory motor set. Selective inhibition, using the central mechanism, might thus be achieved by rapidly invoking such a selective inhibitory set on detection of the stop signal. Predictions for this condition would then be the same as those derived for the stop-change condition.

However, a serious complication in this condition stems from the fact that there are at least two ways in which selective inhibition of the critical response might be accomplished by selectively using the global peripheral mechanism. Participants might initially inhibit all responses when the stop signal occurs, using the fast peripheral mechanism, and subsequently reactivate the GO signal after determining that the response should not be inhibited. Alternatively, they might postpone the inhibitory response until they have determined whether motor inhibition is required and then invoke the fast peripheral mechanism. In principle, these two strategies should be distinguishable from genuinely selective inhibition and from each other on the basis of overt response measures and the LRP. Use of the peripheral mechanism should result in LRP amplitudes on signalinhibit trials that are similar to those in the stop-all condition and considerably larger than those in the stop-change condition. Furthermore, the first strategy should result in short SSRTs, very similar to those in the stop-all condition, but also in greatly slowed primary-task responses on trials where the stop signal was present but the response should not be inhibited. The second strategy, on the other hand, should result in much longer SSRTs. Moreover, as the inhibitory response in this strategy would be contingent upon the outcome of a subset of the component processes in the primary task, the timing of the overt response and the internal inhibitory response would quite likely be positively correlated, thus violating the stochastic-independence assumption of the race model. As demonstrated by De Jong et al. (1990, Appendix), such a violation would cause the race model to seriously underestimate the mean reaction time for trials on which the stop signal was present but the participant failed to withhold the response. Assessment of the adequacy of the fit of the race model might thus provide another useful means of distinguishing between alternative strategies in this condition. 


\section{Method}

\section{Participants}

Eight students were paid $\$ 4$ per hour plus bonuses for participation. The participants (between 18 and 27 years of age) were right-handed and had normal or corrected-to-normal vision and hearing. All participants showed clear lateralization of movementrelated potentials in a short screening session.

\section{Apparatus and Stimuli}

The stimuli for the choice reaction time task were the uppercase letters $M, N, V$, and $W$. Presented on a DEC VT-11 CRT display at a viewing distance of $1 \mathrm{~m}$, the visual angle subtended by each letter was approximately 0.5 degrees. The stop signal was a tone $(1,000$ $\mathrm{Hz}, 50 \mathrm{~ms}$ duration, $65 \mathrm{~dB}$ amplitude), generated by a Schlumberger sine-square audio generator (Model SG-18A) and presented through a speaker placed on top of the display.

Participants responded by squeezing one of two zero-displacement dynamometers with the left or right hand, as a function of the stimulus letter. The system provided a continuous recording of the force output of both hands. A Schmitt trigger could be set to a predetermined force level such that when the exerted force reached this level, an overt "criterion" response was recorded. At the start of the experiment, the participant's maximum force level was determined for each hand. Criterion levels for each hand were then set at $20 \%$ of the maximum force applied by the participant. Participants heard a click when the response force reached criterion during the first two blocks in each session.

\section{Design and Procedure}

The choice task involved classifying single letters from the stimulus set. The letters $V$ and $M$ were assigned to one hand, and the letters $W$ and $N$ to the other. Onset of a central fixation point indicated the start of a trial. The point was extinguished after 500 $\mathrm{ms}$ and followed after another $500 \mathrm{~ms}$ by the presentation of one of the letters. The stimulus letter was extinguished after $1,000 \mathrm{~ms}$ and followed by a $1,500 \mathrm{~ms}$ intertrial interval during which the screen remained blank.

Participants were tested in three sessions on consecutive days and performed 18 blocks of 96 trials in each session. The first session was used for instruction and training. Participants performed the choice task alone in the first two blocks of each session; these blocks were excluded from analysis. The remaining blocks were organized into four miniseries, consisting of four blocks each, in which the four experimental conditions were administered (stop-all, stop-change, selective-left hand, selectiveright hand). The order of the miniseries was randomized across participants, with the restriction that the two miniseries for the selective-stop conditions were always separated by one of the other conditions.

The stop signal occurred on $50 \%$ of the trials in all conditions. In the stop-all condition, the stop signal required participants to withhold responses with either hand. In the stop-change condition, the stop signal required them to withhold responses with either hand and, irrespective of whether the manual response could be inhibited, to make an immediate alternative response by pressing left and right pedals with both feet simultaneously. In the selectivestop condition, the stop signal required them to withhold responses with either the left hand (selective-left) or the right hand (selective-right) but not with the other hand.
Separate stop signal delays were used in the four conditions. All delays were set at $250 \mathrm{~ms}$ at the start of the first session and were then adjusted individually for each participant by a tracking algorithm designed to yield a success rate of inhibition of approximately $50 \%$ in each condition. The tracking algorithm adjusted delays between blocks and was in effect during all sessions. The delay (in ms) for the next block was computed as follows:

$$
\text { Delay }_{\text {next block }}=\text { Delay }_{\text {previous block }}+2 \times\left(\text { rate }_{\text {previous block }}-50\right) \text {, }
$$

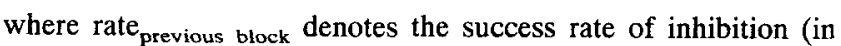
percent) in the previous block. The values at the end of a session served as the initial values for the next session.

The primacy of the choice RT task was emphasized in the instructions. Participants were told to respond quickly while maintaining a high level of accuracy. They were instructed not to delay their responses in anticipation of the stop signal but to make a concerted effort to withhold the response if they detected the stop signal. It was explained to them that they would not always be able to withhold the response and that the computer would in fact adjust the stop signal delay continuously so that they would be able to withhold the response approximately $50 \%$ of the times that the stop signal occurred. A numerical example was used to demonstrate how the strategy of delaying the manual response in anticipation of the stop signal would merely result in ever-longer stop signal delays. The need to make an immediate foot response to the stop signal in the stop-change signal was strongly emphasized.

The order of trials in a block was completely randomized. To reduce practice effects across sessions, the assignment of responses to the two subsets of stimulus letters was reversed between sessions.

\section{Psychophysiological Recording}

The electroencephalogram (EEG) was recorded from $F_{z}, C_{z}, P_{z}$ (according to the $10 / 20$ system), $C_{3}{ }^{\prime}\left(4 \mathrm{~cm}\right.$ to the left of $C_{z}$ ), and $C_{4}{ }^{\prime}$ (4 cm to the right of $C_{z}$ ), referenced to linked mastoids, using $\mathrm{Ag} / \mathrm{AgCl}$ electrodes. Vertical and horizontal electrooculographic activity (EOG) was recorded from locations above and below the right eye and at $2 \mathrm{~cm}$ external to the outer canthus of each eye. Electrode impedance was less than $5 \mathrm{kohm}$ for EEG electrodes and less than $20 \mathrm{kohm}$ for EOG electrodes. The signals were amplified by Grass amplifiers (Model 7P122) and filtered on-line with a high frequency cutoff at $35 \mathrm{~Hz}$ and a time constant of $8 \mathrm{~s}$. The signals were digitized at $100 \mathrm{~Hz}$ for $2,100 \mathrm{~ms}$, starting $100 \mathrm{~ms}$ before the onset of the fixation point.

\section{Data Analysis}

An informal explanation of the race-model analyses has been provided earlier (see Figure 1); here we describe the procedure more formally. The RTs on no-signal trials were rank ordered, and the $n$th fastest time was determined, where $n$ is the number of no-signal trials multiplied by the probability of responding when the stop signal occurred. A predicted value for mean signalrespond RT was then computed by averaging the $n$ fastest nosignal RTs. The $n$th value also provides an estimate of the time of occurrence of the inhibitory response to the stop signal, relative to the onset of the primary-task stimulus. An estimate of SSRT was obtained by subtracting out the stop-signal delay. Because the stop-signal delay differed between blocks, this analysis was performed separately for every trial block, and the predicted and estimated values were then averaged across blocks. For the selective-stop condition, the no-signal trials used in this analysis were 
those for which the response was made by the critical hand (e.g., left-hand responses on no-signal trials in the selective-left condition).

For subsequent analyses, we used an algorithm, described in detail in De Jong et al. (1990), to detect partial responses, which are defined as subcriterion overt responses on signal-inhibit trials. The number of partial responses was too small (less than 10 per condition) for most participants to permit meaningful analysis of these responses. The use of the LRP amplitude on signal-inhibit trials in order to distinguish between central and peripheral means of motor inhibition required that analysis of signal-inhibit trials be restricted to trials on which no overt movement at all took place; partial responses were therefore excluded from subsequent analyses.

The EEG signals were inspected for artifacts, corrected off-line for vertical and horizontal eye movements and blinks by standard procedures (Gratton, Coles, \& Donchin, 1983), and then smoothed with a low-pass digital filter (high-frequency cutoff at $8.0 \mathrm{~Hz}$ ). Trials were categorized into four categories: (a) signal-respond trials, (b) no-signal trials corresponding, according to the race model, to signal-respond trials (see Figure 1), (c) signal-inhibit trials, and (d) no-signal trials corresponding to signal-inhibit trials (De Jong et al., 1990). The LRP was computed for each trial category by computing separate average $C_{3}{ }^{\prime}-C_{4}{ }^{\prime}$ difference potentials for left-hand and for right-hand responses, followed by subtracting the difference potential for left-hand responses from that for right-hand responses (Coles, 1989; De Jong, Wierda, Mulder, \& Mulder, 1988).

\section{Results and Discussion}

\section{$R T s$}

Mean no-signal RTs, stop-signal delays, percentages of signal-respond trials, and RTs for signal-respond trials for each condition are presented in Table 1 . Mean signalrespond RTs as predicted by the race model and estimated SSRTs are also presented. These results will be discussed for each condition in turn.

Stop-all. The difference between observed and predicted values for mean signal-respond RT did not reach significance, $F(1,7)=4.52, p>.07$, indicating a reasonably good fit of the race model for this condition. This

\section{Table 1}

Experiment 1: Mean No-Signal RTs, Stop-Signal Delays, Percentages of Signal-Respond Trials, Observed and Predicted Signal-Respond RTs, and Estimated Stop-Signal RTs

\begin{tabular}{lccc}
\hline & \multicolumn{3}{c}{ Condition } \\
\cline { 2 - 4 } \multicolumn{1}{c}{ Variable } & Stop-all & Stop-change & Selective-stop \\
\hline No-signal RT & 443 & 455 & 429 \\
Stop-signal delay & 267 & 237 & 182 \\
\% signal-respond trials & 51.9 & 51.5 & 49.1 \\
Signal-respond RTs & & & \\
$\quad$ Observed & 400 & 411 & 434 \\
$\quad$ Predicted & 394 & 410 & 407 \\
Estimated stop-signal RT & 174 & 220 & 273 \\
\hline
\end{tabular}

Note. All times are in milliseconds. $\mathrm{RT}=$ reaction time. result, as well as the fast estimated SSRT, replicates previous findings for this condition (e.g., De Jong et al., 1990).

Stop-change. The difference between observed and predicted values for mean signal-respond RT did not approach significance $(F<1)$, indicating an excellent fit of the race model for this condition. The estimated SSRT was significantly longer than that in the stop-all condition (220 versus $174 \mathrm{~ms}$, respectively; $F(1,7)=70.44, p<.001$ ). This result is consistent with the possibility that inhibition was accomplished by the slower central selective inhibitory mechanism in the stop-change condition. As we noted earlier, however, it might also be because the stop signal required not only inhibition of the manual response but also the swift execution of the alternative foot response. Mean no-signal RTs in the stop-all and stop-change conditions did not differ, $F(1,7)=1.50, p>.25$, indicating that the additional requirement to make a fast foot response to a possible stop signal did not affect performance of the primary task.

Mean RT for the foot response to the stop signal was computed separately for signal-inhibit and signal-respond trials. The RTs were 285 and $298 \mathrm{~ms}$ for signal-inhibit and signal-respond trials, respectively; the difference did not reach significance, $F(1,7)=3.46, p>.1$. This result differs from that reported by Logan and Burkell (1986; see also Logan, 1985), who found much longer RTs for the response to the stop signal when participants failed to inhibit the primary-task response. Possible reasons for these different results will be discussed later.

Selective-stop. The race model significantly underestimated the mean signal-respond RT by $27 \mathrm{~ms}, F(1,7)=$ $12.3, p<.01$. The simulation results reported by De Jong et al. (1990) indicate that a poor fit of this type might reflect a violation of the stochastic-independence assumption of the race model. It is important to note that those simulation results also indicate that such a violation would cause the race-model analysis procedure to underestimate SSRT for this condition. Despite the fact that the estimated SSRT in the selective-stop condition might underestimate the "true" value, it was nevertheless substantially longer than that in the stop-change condition $(273$ versus $220 \mathrm{~ms}$, respectively; $F(1,7)=16.12, p<.01)$.

Further insight into how motor inhibition was accomplished in the selective-stop condition can be gained by comparing the mean RTs for the following three trial categories: (a) no-signal trials where the required response was on the noncritical hand ( $429 \mathrm{~ms})$, (b) no-signal trials where the response was on the critical hand $(466 \mathrm{~ms})$, and (c) signal trials where the required response was on the noncritical hand $(473 \mathrm{~ms})$. The RT difference between the first two categories was significant, $F(1,7)=6.69, p<.04$, indicating that participants tended to delay responses with the critical hand to enhance their chances of being able to withhold these responses when necessary, despite our efforts to prevent this strategy. The difference between the first and third categories was highly significant, $F(1,7)=$ $73.49, p<.001$, indicating that the presence of the stop signal consistently slowed processing in the primary task, even when the response did not in fact need to be inhibited. 
These results for the selective-stop condition permit some preliminary conclusions. The fact that the SSRT in this condition was much longer than that in the stop-all condition argues against the possibility that selective inhibition was accomplished by first invoking the fast peripheral mechanism and then reactivating the response in case it should not be inhibited. Why then were responses with the noncritical hand slower in the presence of the stop signal (by an average of $44 \mathrm{~ms}$ )? One possibility is that participants may have used the former strategy some of the time. But it is also possible that this effect reflects interference with primary-task processing caused by the processing of the stop signal. For instance, the decision as to whether the peripheral inhibition mechanism should be invoked may well have interfered with decisional processes in the primary task.

Such interfering effects of stop-signal processing on primary-task performance would constitute violations of the signal-independence assumption of the race model, according to which primary-task performance should remain unaffected by the presence of the stop signal. It is important to note that violations of signal independence can also readily explain why the race model underestimated mean signalrespond RT, as this estimate was based on no-signal trials where primary-task processing would proceed without interference. These considerations imply that our initial inference, that the poor fit of the race model in the selective-stop condition was due to a violation of stochastic independence and thus indicative of a contingent use of the peripheral inhibition mechanism, might not be valid. As a consequence, the behavioral data, while ruling out one possible strategy, also appear to be neutral with respect to whether motor inhibition in the selective-stop condition was achieved by using the central or the peripheral mechanism.

\section{$L R P s$}

LRPs were computed for signal-inhibit trials without any overt movement, for signal-respond trials, and for no-signal trials from the regions of the no-signal RT distribution that correspond, according to the race model, to the signalinhibit and signal-respond trials (see De Jong et al., 1990). The four LRP waveforms, for each condition, averaged across participants are shown in Figure 2.

Several aspects of these waveforms are noteworthy. First, the waveforms for signal-respond trials and the corresponding no-signal trials overlap almost perfectly in the stop-all and the stop-change conditions, suggesting that central response-activation processes remained virtually unaffected by the processing of the stop signal on signal-respond trials in these conditions. The close overlap in the stop-change condition demonstrates that the ability of the LRP to reflect central activation of the manual response was not affected by the temporally overlapping activation and execution of the foot response, in accordance with the results of Osman and Moore (1993). The LRPs in the selective-stop condition show some evidence of interference by the processing
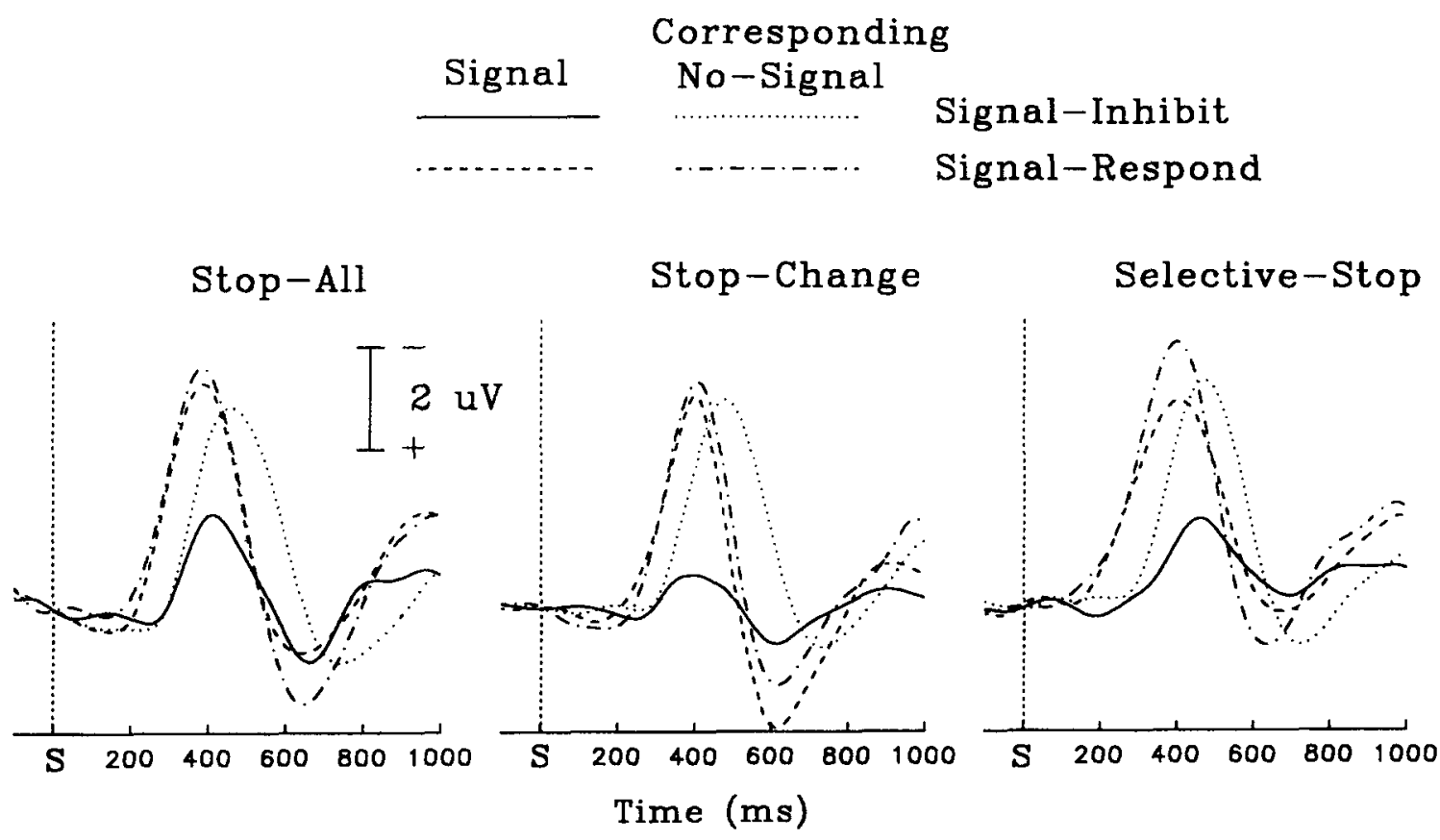

Figure 2. Waveforms of the lateralized readiness potential (LRP) for signal-respond and signalinhibit trials, and for the corresponding no-signal trials. Upward (negative) deflections indicate a greater negativity over the hemisphere contralateral to the correct response hand. S denotes the onset of the primary task stimulus. Stop-all condition (left), stop-change condition (middle), and selectivestop condition (right). $\mathrm{uV}=$ microvolt. 
of the stop signal on central response activation on signalrespond trials, consistent with the behavioral results.

The most important results for present purposes concern the maximum amplitude reached by the LRP on signalinhibit trials. Figure 2 shows that this amplitude was considerable in the stop-all condition, replicating our previous findings (De Jong et al., 1990), and in the selective-stop condition, but it was much smaller in the stop-change condition. A combined Wilcoxon/t-test procedure (for details, see De Jong et al., 1988, 1990) revealed that the partial LRPs for signal-inhibit trials reached statistically significant amplitudes in all three conditions $(p s<.0001$ in conditions stop-all and selective-stop, $p<.03$ in condition stop-change). In all three conditions, therefore, central response activation often reached appreciable levels in the absence of any overt movement.

Comparison of the LRP waveforms for signal-inhibit with corresponding no-signal trials in Figure 2 shows that central response-activation processes were subject to inhibition on signal-inhibit trials; this is clear evidence that a central inhibition mechanism was operative in all three conditions. A crucial question is whether this mechanism was in fact primarily responsible for preventing any overt movement on these trials. To address this question, we compared the maximum LRP amplitude on signal-inhibit trials with the mean LRP amplitude at time of squeeze onset on corresponding no-signal trials. The rationale for this comparison is based on previous findings that the average LRP amplitude at time of onset of muscle activity (EMG) or overt movement remains fixed across experimental conditions and different RT bins (De Jong et al., 1990; Gratton, Coles, Sirevaag, Eriksen, \& Donchin, 1988). This invariant relation between LRP amplitude and movement onset suggests that central motor commands tend to be issued when the difference between the central activation levels of the two hands reaches a relatively fixed criterion level or threshold. ${ }^{1}$ As discussed earlier, the central inhibition mechanism, which leaves the GO signal unaffected, can prevent overt movement only by preventing the difference between central activation levels from reaching this threshold. Consequently, the maximum LRP amplitude for successfully stopped responses (signal-inhibit trials) should in that case remain well below the threshold amplitude. No such requirement exists when the peripheral mechanism is used to inhibit the GO signal, so that the LRP on signal-inhibit trials should be able to reach or exceed the threshold level.

The mean LRP amplitude at squeeze onset provides a measure of the threshold for central motor outflow. However, this measure overestimates the actual value of the threshold because it does not take into account the transmission lag for central motor commands to result in overt movement. From transcranial stimulation studies of the corticospinal system in humans and biomechanical properties of muscles, this lag can be estimated to be at least $50 \mathrm{~ms}$ for the squeeze responses used here (Benecke, Meyer, Gohmann, \& Conrad, 1988; De Jong et al., 1990). The mean LRP amplitude at $50 \mathrm{~ms}$ before squeeze onset was therefore used as an additional, and more accurate, measure of the threshold value.

The results of this analysis, averaged across subjects, are shown in Table 2. There were no significant differences between the mean LRP amplitudes for no-signal trials corresponding to signal-respond and for those corresponding to signal-inhibit trials (all $p \mathrm{~s}>.20$ ); this confirms earlier findings that the LRP amplitude at response onset does not vary as a function of relative response speed (De Jong et al., 1990; Gratton et al., 1988). Despite this equivalence, comparisons between maximum and mean LRP amplitudes were restricted to signal-inhibit trials and corresponding no-signal trials in order to maintain consistency with the race-model framework of analysis. For the stop-all and selective-stop conditions, the mean maximum LRP amplitude on signal-inhibit trials approximated the mean LRP amplitude at squeeze onset and substantially exceeded the mean LRP amplitude $50 \mathrm{~ms}$ before squeeze onset. For the stop-change condition, in contrast, the maximum LRP amplitude on signal-inhibit trials was considerably smaller than the mean LRP amplitude both at squeeze onset and at $50 \mathrm{~ms}$ before squeeze onset. A two-factor repeated-measures analysis of variance (ANOVA) was conducted to compare the maximum LRP amplitudes on signal-inhibit trials with the mean LRP amplitudes $50 \mathrm{~ms}$ before squeeze onset as a function of experimental condition (first and third rows of Table 2). The analysis yielded a significant main effect of condition, $F(2,14)=10.80, p<.001$, no overall difference between maximum and mean amplitudes $(F<$ $1)$, and a significant interaction between these factors, $F(2,14)=11.97, p<.001$.

These results indicate that the normal association between central response activation and overt movement was disrupted on signal-inhibit trials in the stop-all and selectivestop conditions. This suggests that the peripheral inhibition mechanism was primarily responsible for motor inhibition in these conditions, by preventing the execution of central motor commands. No such disruption was evident for the stop-change condition, in which the LRP amplitude on signal-inhibit trials remained well below criterion levels associated with overt movement. This provides evidence that motor inhibition in this condition was achieved primarily by the central inhibition mechanism, by selectively preventing central outflow of motor commands.

\footnotetext{
${ }^{1}$ Consistent with this idea, Cohen, Servan-Schreiber, and McClelland (1992) found in a connectionist simulation study a strong correlation between response emission and the difference between activation levels of the two hands. Such a correlation occurred despite the fact that in their model a response was emitted when the activation of one hand reached a fixed threshold, regardless of the (subthreshold) level of activation of the other hand. This correlation was a consequence of reciprocal inhibitory interactions between the central representations of the two hands. We also note that recent evidence suggests that the threshold for response emission may not be entirely fixed but may decrease with extended practice and vary as a function of speed-accuracy instructions (Smid, 1993).
} 
Table 2

Experiment 1: Mean Maximum LRP Amplitudes for Signal-Inhibit Trials and Mean LRP Amplitudes at Squeeze Onset and at $50 \mathrm{~ms}$ Before Squeeze Onset for No-Signal Trials Corresponding to Signal-Inhibit and Signal-Respond Trials

\begin{tabular}{cccc}
\hline & \multicolumn{3}{c}{ Condition } \\
\cline { 2 - 4 } Trial & Stop-all Stop-change Selective-stop \\
\hline $\begin{array}{c}\text { Maximum LRP amplitude: } \\
\quad \text { signal-inhibit }\end{array}$ & 2.78 & 1.21 & 2.59 \\
$\begin{array}{c}\text { Mean LRP amplitude: no- } \\
\quad \begin{array}{l}\text { signal corresponding } \\
\text { to signal-inhibit }\end{array}\end{array}$ & 3.06 & 2.99 & 3.27 \\
$\begin{array}{c}\text { At squeeze onset } \\
50 \text { ms before squeeze } \\
\quad \text { onset }\end{array}$ & 1.87 & 1.91 & 2.04 \\
$\begin{array}{c}\text { Mean LRP amplitude: no- } \\
\text { signal corresponding } \\
\quad \text { to signal-respond }\end{array}$ & & & \\
$\begin{array}{c}\text { At squeeze onset } \\
\text { 50 ms before squeeze } \\
\text { onset }\end{array}$ & 2.94 & 3.05 & 3.11 \\
\hline
\end{tabular}

Note. Amplitudes are in microvolts. LRP $=$ lateralized readiness potential.

\section{Further Discussion}

The results in the stop-all condition closely replicated previous results with the same condition, demonstrating fast motor inhibition and a dissociation on signal-inhibit trials between central response activation, as measured by the LRP, and overt movement. This replication by itself does not, of course, strengthen the case for the hypothesis that motor inhibition can be achieved through employment of either a relatively slow central mechanism or a faster peripheral mechanism, as these results constituted in fact the original basis for this hypothesis (De Jong et al., 1990). The stop-change condition provided a direct test of the twomechanism hypothesis, as the requirement to make a swift alternative response to the stop signal should necessitate the use of the central mechanism to selectively inhibit the manual response. We therefore predicted slower motor inhibition and LRP amplitudes on signal-inhibit trials that remain well below criterion levels associated with overt movement. The data confirmed both predictions, thereby providing compelling support for the hypothesis.

The foot response to the stop signal was found to be only slightly slower when inhibition of the manual response failed. Thus, the manual and foot responses seem to have been executed with a high degree of independence. ${ }^{2}$ The same conclusion is suggested by the fact that the LRP, which reflects the central activation of the manual response, was not affected by the overlapping activation and execution of the foot response on signal-respond trials. As noted earlier, these results differ from those reported by Logan (1985) and Logan and Burkell (1986), who used a manual alternative response to the stop signal and found that this response was considerably delayed when participants failed to inhibit the manual response in the primary task. Similar differences in interference between manual-manual and manual-foot response combinations have been found in the overlapping-task paradigm by De Jong (1993). Such differences were taken to indicate that limitations in overlapping task performance are caused in part by a response-initiation bottleneck that prevents two independent, discrete responses from being initiated in close succession (De Jong, 1993; Logan \& Burkell, 1986). To account for responsemodality effects, the response-initiation bottleneck was interpreted to reflect a modality-specific functional coupling between effector systems that facilitates their coordination, as in performing bimanual acts, but also causes interference or cross-talk when the systems need to operate independently (De Jong, 1993). Such modality-specific coupling might result if GO signals for different modalities were generated by separate peripheral movement-energizing systems. However, the present results argue against this possibility, as they indicate that the same GO signal can energize both hand and foot movements. This suggests that modalityspecific cooperative and competitive motoric interactions stem from cooperation and cross-talk between simultaneously active control processes at more central levels in the motor system (Swinnen, 1992). Further implications of this view will be discussed later.

The analysis of performance in the selective-stop condition was complicated by the possibility of different strategies that involved either the central or the peripheral inhibition mechanism. The behavioral results were found on closer analysis to be neutral regarding the question of whether selective inhibition was achieved by the central mechanism or by invoking the peripheral mechanism after determining that the response should be inhibited. This uncertainty was resolved by the finding that the LRP amplitude on signal-inhibit trials exceeded levels normally associated with overt movement, suggesting that motor inhibition in the selective-stop condition was effected by the peripheral mechanism.

The latter inference should be regarded as tentative, however, as it is based solely on the assumption that the comparison between the mean LRP amplitude at movement onset and the maximum LRP amplitude on signal-inhibit trials provides a valid diagnostic measure for distinguishing between central and peripheral means of motor inhibition. Though this assumption received support from the fact that the predicted differences between the LRP amplitudes on signal-inhibit trials in the stop-all and stop-change conditions were in fact obtained, converging evidence is needed before the inference can be accepted with reasonable confidence. Experiment 2 was therefore designed to put this inference to a further and independent test. If motor inhibition in the selective-stop condition is indeed accomplished

\footnotetext{
${ }^{2}$ It also seems likely that the time to process the stop signal is not constant but subject to some random variation. A failure to inhibit would then be due in part to relatively slow stop-signal processing and would thus tend to be associated with a slower foot response. This, rather than any lack of independence between the manual and foot responses, might have been responsible for the slightly slower foot responses on signal-respond trials.
} 
by invoking the peripheral mechanism after determining that response inhibition is required, then any primary-task manipulation that affects the time at which this determination can be made should similarly affect the SSRT. Such effects on SSRT would not be expected if selective inhibition is accomplished by the central selective mechanism, as the activation of this mechanism should be contingent only on the detection of the stop signal and not on the completion of component processes for the primary task.

\section{Experiment 2}

Motor inhibition in the selective-stop condition was first studied by Logan et al. (1986). They performed two experiments with different primary tasks. One task used letter stimuli that were assigned randomly to the response keys. The other task used Xs presented in four different locations that were assigned to spatially compatible response keys. Primary-task responses were much faster for the latter task, but the SSRTs in the two experiments were similar. These results rule out the possibility that participants postponed the inhibitory response until the required primary-task response had been determined. However, the possibility remains that the inhibitory response might be contingent on primary-task processing in some other fashion. In particular, because the stimulus-response mapping in either task was fixed, the decision to elicit the inhibitory response might be based on the identity of the primary-task stimulus rather than that of its associated response; that is, participants might recognize and use the fact that a response to the stop signal is required only for a critical subset of primary-task stimuli. In that case, there would be no strong reasons to expect different SSRTs for primary tasks of different stimulus-response compatibility, as compatibility effects are generally believed to be primarily located in postperceptual processing stages (Kornblum, Hasbroucq, \& Osman, 1990). Thus, the results of Logan et al. (1986) are not necessarily inconsistent with the preliminary conclusion derived on the basis of the LRP results for the selective-stop condition in Experiment 1.

To examine this issue further, we used the same primary task as in Experiment 1 and manipulated stimulus discriminability by overlapping the stimulus letter with a noise mask on half of the trials. This manipulation was chosen because it should primarily affect relatively early perceptual processes and thus prolong the finishing times of stimulus identification, response selection, and the RT to approximately the same extent. From the results for the selectivestop condition in Experiment 1, we predicted that this manipulation would have equivalent effects on SSRT. Note that this prediction should hold whether the activation of the peripheral inhibitory mechanism is contingent on stimulus identification or on response selection.

Special measures were taken to discourage participants from strategically slowing the critical response in order to enhance their chances of being able to inhibit the response when required. Despite our efforts to prevent this strategy in Experiment 1, the results showed that some such slowing occurred in that experiment, demonstrating that people are able to adopt a tonic selective inhibitory motor set. However, this ability is not particularly surprising. At issue in the present research is whether and how people can rapidly and selectively inhibit motor responses when the need to do so arises unexpectedly. The presence of a superimposed tonic inhibitory motor set would undoubtedly complicate the investigation of phasic motor inhibition. Although interactions between tonic and phasic inhibitory mechanisms might prove to be an interesting topic for further study, it seems desirable at this point to avoid such complications.

\section{Method}

\section{Participants}

Ten undergraduate students participated in partial fulfillment of a course requirement.

\section{Apparatus and Stimuli}

The stimuli were identical to those in Experiment 1. The stimulus letters were presented in white on black on a Seiko CM-1440 color monitor controlled by an IBM compatible microcomputer (equipped with VGA graphics, providing a display resolution of $640 \times 480$ pixels). A new visual noise mask was constructed for every noise trial by turning on a random $25 \%$ of the pixels in a $30 \times 30$ pixel grid centered on and completely containing the letter. A $1,000 \mathrm{~Hz}$ tone served as the stop signal and was presented through the speaker of the microcomputer. The $Z$ and "P" keys of the computer keyboard served as response keys, to be pushed with the left and right digits, respectively.

\section{Design}

Participants served in three sessions of about $1 \mathrm{hr}$; the first session was for training only. Each session consisted of 18 blocks of 60 trials. Participants performed the choice task alone in the first two blocks of each session; these blocks were excluded from analysis. The remaining blocks were organized into four miniseries, consisting of four blocks in which the critical hand (left or right) was kept fixed. The critical hand was switched between miniseries; half of the participants started with the left hand and the other half with the right hand as the critical hand.

The stop signal occurred on $50 \%$ of the trials. The same stop signal delay was used for intact and degraded stimuli. The stop signal delay was adjusted between blocks, using the same algorithm as in Experiment 1, to yield an overall success rate of inhibition of approximately $50 \%$. The letter stimulus was overlapped by the noise mask on $50 \%$ of the trials. The order of trials in a block was completely randomized. The assignment of the two subsets of stimulus letters to the responses was reversed between sessions in order to reduce practice effects across sessions.

The instructions were the same as for the selective-stop condition in Experiment 1. Special precautions were taken to discourage participants from delaying responses with the critical hand. At the end of each block, separate mean RTs were computed for responses with the two hands on no-signal trials, and a high-pitch tone and flashing warning message were presented for $30 \mathrm{~s}$ if the mean RT for the critical hand exceeded that for the noncritical 
hand by $60 \mathrm{~ms}$ or more. This annoying feedback proved very effective in preventing strategic slowing of the critical response.

\section{Results and Discussion}

Mean no-signal RTs, stop-signal delays, percentages of signal-respond trials, and RTs for signal-respond trials are presented in Table 3 for normal (high discriminability) and degraded (low discriminability) stimuli. Also presented are the mean predicted signal-respond RTs and the estimated SSRTs.

An ANOVA of the no-signal RTs yielded only a highly significant effect of discriminability, $F(1,9)=272.24, p<$ .001 . The RT difference between the critical and noncritical hand was not significant $(F<1)$, indicating that our attempts to dissuade our participants from slowing responses with the critical hand were quite successful. Mean RTs for signal trials when the response was on the noncritical hand were 469 and $547 \mathrm{~ms}$ for normal and degraded stimuli, respectively. An ANOVA in which these values were compared with the corresponding no-signal RTs for responses with the noncritical hand yielded a significant main effect of stop-signal presence, $F(1,9)=44.00, p<.001$, and an interaction of this effect with discriminability, $F(1,9)=$ $7.28, p<.03$. Thus, the processing of the stop signal interfered with primary-task performance, even when the response should not be inhibited, and the detrimental effect of such interference was stronger when the primary-task stimulus was degraded.

With the same stop-signal delay, the success rate of inhibition was considerably higher for degraded than for normal stimuli, $F(1,9)=32.12, p<.001$. As in Experiment 1 , the fit of the race model was rather poor, as evidenced by a significant deviation between observed and predicted mean signal-respond RTs, $F(1,9)=9.58, p<.02$. Though this deviation appears to be somewhat larger for degraded stimuli, the relevant interaction did not approach significance, $F(1,9)=2.32, p>.16$. Finally, and most important, the estimated SSRT was significantly longer (by $41 \mathrm{~ms}$ ) for degraded stimuli, $F(1,9)=18.72, p<.002$.

\section{Table 3}

Experiment 2: Mean No-Signal Reaction Times (RTs), Stop-Signal Delays, Percentages of Signal-Respond Trials, Observed and Predicted Signal-Respond $R T$, and Estimated Stop-Signal RTs

\begin{tabular}{lcc}
\hline & \multicolumn{2}{c}{ Discriminability } \\
\cline { 3 - 3 } Variable & High & Low \\
\hline No-signal RT & & \\
$\quad$ Noncritical hand & 444 & 507 \\
Critical hand & 449 & 514 \\
Stop-signal delay & 158 & 158 \\
\% signal-respond trials & 63.1 & 48.3 \\
Signal-respond RTs & & \\
Observed & 407 & 471 \\
Predicted & 395 & 443 \\
Estimated stop-signal RT & 304 & 345 \\
\hline
\end{tabular}

Note. All times are in milliseconds.
As in the selective-stop condition of Experiment 1, the present data also indicate that processing of the stop signal interfered with primary-task performance, thus violating the signal-independence assumption of the race model. The predicted signal-respond RT was based on no-signal trials where no such interference was present and would therefore almost certainly be too small. The present data provided a unique opportunity to estimate and take account of the effects of signal dependencies by basing the prediction of signal-respond RT not on no-signal trials but on signal trials where the same dependencies must have been operative but the required response was on the noncritical hand. This analysis was possible because, unlike in Experiment 1, participants did not seem to delay responses with the critical hand as compared with those with the noncritical hand. The predicted mean signal-respond RTs in this analysis were 408 and $461 \mathrm{~ms}$ for normal and degraded stimuli, respectively. These predicted values did not differ significantly from the observed values $(F<1)$, indicating that the race model was able to fit the data very well after the effects of the violation of signal independence had been incorporated. This suggests that the two other basic tenets of the model, that response activation and inhibition processes are involved in a race and operate in a stochastically independent fashion, provide an adequate description of performance in the selective-stop condition. The estimated SSRTs for normal and degraded stimuli in this analysis were 325 and 375 $\mathrm{ms}$, respectively. The $50 \mathrm{~ms}$ discriminability effect on SSRT was highly significant, $F(1,9)=20.0, p<.002$. The effects of discriminability on SSRT and on no-signal RTs were not significantly different, $F(1,9)=1.23, p>.29$.

\section{Further Discussion}

The effect of stimulus degradation on primary-task RT (64 $\mathrm{ms}$ ) was almost fully reflected in the time required to selectively inhibit responses $(50 \mathrm{~ms})$. This finding indicates that selective inhibition in the present experiment was accomplished primarily, though perhaps not exclusively, by activating the global peripheral mechanism after determining that motor inhibition was required. If selective inhibition operated by activating a genuinely selective inhibitory motor set on detection of the stop signal, using the central inhibition mechanism, then stimulus degradation should have had no effect on the time required to inhibit responses. The present results taken by themselves are ambiguous as to whether the determination that inhibition was required was contingent on stimulus identification or on response selection in the primary task. However, the former possibility should be considered more likely considering that manipulations of stimulus-response compatibility have been found to have little or no effect on SSRT (Logan et al., 1986).

The results of the adapted race-model analysis suggested no significant violations of stochastic independence after the effects of signal dependencies had been taken into account. This might seem surprising because a contingency of motor inhibition on identification of the primary-task 
stimulus would seem to provide good reasons to expect a positive correlation between the completion times of response activation and inhibition processes. Evidence that the variance in the time needed to identify familiar, discrete stimuli makes a relatively minor contribution to the overall variance in the finishing times of response selection and activation processes (De Jong et al., 1988), provides a resolution to this puzzle; in that case, the positive dependency, and its effect on the fit of the race model, should be expected to be small and difficult to detect.

Finally, it is important to note that the present results are entirely consistent with, and were indeed predicted from, our interpretation of the LRP results for the selective-stop condition in Experiment 1. This provides strong converging evidence that the comparison between mean LRP amplitude at movement onset and maximum LRP amplitude on signal-inhibit trials represents a useful and valid diagnostic tool for distinguishing between central and peripheral means of motor inhibition.

\section{General Discussion}

The results of this study provide a reasonably coherent picture of the mechanisms and strategies of inhibitory motor control in a variety of situations. On the basis of earlier findings, we postulated that two different mechanisms can effect inhibition of planned or ongoing movements. One mechanism operates at a peripheral level of the motor system and prevents the overt expression of any motor commands issued by more central levels by inhibiting the peripherally generated GO signal that is needed to energize the movement. This mechanism results in fast inhibition and is capable of preventing overt movement even when outflow of central motor commands cannot be prevented. However, its global mode of operation limits the use of this mechanism to situations where motor inhibition can be all-or-none. The second, centrally operating mechanism effects motor inhibition by inhibiting central motor-activation processes while leaving the GO signal intact. This mechanism leads to less rapid inhibition but has an important advantage in that it can selectively inhibit some responses without impeding the implementation and execution of concurrent or alternative movements, making this mechanism seem the obvious choice in situations where selective motor inhibition is required.

The results for the stop-all condition, which required all-or-none motor inhibition, and the stop-change condition, which required simultaneous inhibition of the manual response and execution of an alternative foot response, supported the two-mechanism hypothesis. The additional requirement in the latter condition to execute an alternative response to the stop signal itself should prevent the use of the peripheral mechanism and make motor inhibition dependent solely on the use of the slower central mechanism. Accordingly, motor inhibition was found to operate more slowly in the stop-change condition. Also, and contrary to what was observed in the stop-all condition, central response activation, as measured by the LRP, was found to remain well below levels normally associated with overt movement when the manual response was successfully inhibited in the stop-change condition. Although evidence for the operation of a central mechanism for motor inhibition was found in both the stop-all and the stop-change conditions, only in the latter condition did this mechanism play a critical role in determining actual success in withholding the response.

The results for the selective-stop condition were also consistent with the two-mechanism hypothesis, but demonstrating this involved two additional steps. The fact that the LRP on signal-inhibit trials exceeded criterion amplitudes normally associated with onset of overt movement suggested the critical involvement of the peripheral mechanism in motor inhibition in this condition. In conjunction with the behavioral results, this led us to conclude tentatively that selective motor inhibition in this condition was not achieved by the central selective mechanism but by activating the global peripheral mechanism after determining that inhibition was required. This inference received strong confirmation from the finding in Experiment 2 that manipulation of the time at which this determination could be made, by manipulation of stimulus discriminability, resulted in almost equivalent effects on the speed of primary-task processing and the timing of motor inhibition.

These results have two important implications. First, they put the theoretical distinction between two functionally distinct motor inhibition mechanisms, one operating at a central level and the other at a more peripheral level, on a solid empirical footing. In combination with the computational approach to motor control developed by Bullock and Grossberg $(1988,1991)$, this theoretical perspective should provide a fertile basis for future investigations of the nature and relative roles of motor activation and inhibition processes in adaptive motor control. Second, these results provide compelling evidence that the comparison between the maximum LRP amplitudes on signal-inhibit trials and criterion amplitudes for movement onset provides a valid and useful tool to discriminate between centrally and peripherally operating mechanisms for inhibitory control of manual responses. An interesting question for future research concerns the probable neuroanatomical loci of these mechanisms (see Bullock \& Grossberg, 1991, for some suggestions). The present evidence only indicates that the central mechanism operates "upstream" and the peripheral mechanism "downstream" from the main generator of the LRP, the primary motor cortex (Coles, 1989).

\section{Changing Planned or Ongoing Movements}

It is of interest to compare the results in the stop-change condition to those obtained in the classic double-step paradigm that has also been used to study the ability to rapidly replace one movement by another. In the double-step paradigm, the target for the movement is occasionally displaced just before or just after the movement to the initial target position has started. Though it has been used mainly with saccadic eye movements (e.g., Becker \& Jurgens, 
1979), we will focus here on studies that have used hand movements (Georgopoulos, Kalaska, \& Massey, 1981; Soechting \& Lacquaniti, 1983). The general finding in these studies is that the modification of the movement induced by the change in target position proceeds in a continuous fashion, with the movement to the original position being gradually substituted by the movement to the new position. This suggests that the modification was accomplished centrally, by substitution of the original movement goal by the new one, while leaving the GO signal intact. This inference is supported by the finding by Georgopoulos et al. (1981) that the peak velocity attained on the way to the new position was generally much higher than that of the control (no-change) movement. Bullock and Grossberg (1988) were able to explain this phenomenon by assuming that the GO signal builds up continuously in time after onset of the original target. The movement to the new position can then proceed more quickly because it is driven by a GO signal that is already large at the time this movement gets underway. It thus appears that rapid movement changes are accomplished at a central level by substituting one goal for another, whether the new movement is made by the same effector system, as in the double-step paradigm, or by a different one, as in the stop-change condition.

The results for the stop-change condition support the notion that the peripherally generated GO signal operates in a largely nonspecific fashion, simultaneously energizing any movements issued by the central motor system. This design automatically constrains muscle synergists participating in a movement or movements by different effector systems participating in an action to function in a synchronous fashion. This synchronization tendency is evident not only in cyclical and noncyclical two-handed movements (Kelso, Putnam, \& Goodman, 1983; Swinnen, 1992), but it applies also to combinations of upper- and lower-limb movements (Baldissera, Cavallari, \& Civaschi, 1983) and of hand movements and speech (Chang \& Hammond, 1987). ${ }^{3}$ On the positive side, this tendency ensures smooth and straight movements (Bullock \& Grossberg, 1988) and reduces the computational complexity of coordinating multilimb actions (Swinnen, 1992). On the negative side, it imposes limitations on the differentiation of action patterns that require component movements to proceed asynchronously, as in playing a musical instrument. The present perspective suggests that such differentiation must be realized at central motor levels, by regulating the timing of outflow of central motor commands.

Modality-specific cooperative and competitive motoric interactions are mediated by cooperation and cross-talk between central representations of movements by similar effector systems, such as the upper limbs. Such interactions induce assimilation between temporally overlapping movements of each others' structural or topological characteristics, such as direction and extent (Newell, 1985). Automatic assimilation will facilitate motor coordination if the movements share many structural characteristics but will hamper performance if the movements are structurally different or need to be executed independently. Consistent with the presumed modality-specific nature of central assimilation tendencies, foot responses in the stop-change condition were found to be executed independently of temporally overlapping manual responses, whereas strong interference has been found between temporally overlapping but independent manual responses (De Jong, 1993; Logan \& Burkell, 1986).

\section{Selective Inhibitory Motor Sets}

Why was selective inhibition of responses by one of the hands not accomplished by activating a genuinely selective inhibitory motor set on detection of the stop signal? It is not the case that such selective sets cannot be attained, because participants exhibited a strong tendency to use such sets in a tonic fashion to enhance their ability to selectively inhibit responses. It might be that the rapid activation of a selective inhibitory set was a possible strategy but that most participants opted to use a different strategy instead. Another possibility is that the construction and activation of selective inhibitory motor sets is a relatively complex process that cannot be invoked rapidly enough to meet the requirements in the selective-stop condition. An important reason for this difficulty may be that the two hands are functionally coupled effector systems, so that inhibiting one but not the other might require special and time-consuming procedures to counteract automatic assimilation between the two hands (Heuer, 1993). If this is indeed the case, then we may expect to find evidence for rapid selective inhibition by genuinely selective means when the alternative responses are made by effector systems that are not functionally coupled, such as hand and feet. That issue is currently under investigation.

\footnotetext{
${ }^{3}$ Saccadic eye movements represent a special case, as these movements do not appear to be synchronized with other movements and can be executed without interference while carrying out a concurrent motor task (Pashler, Carrier, \& Hoffman, 1993). This is consistent with the fact that oculomotor responses are controlled by separate and highly specialized brain structures (Schiller, Sandell, \& Maunsell, 1987).
}

\section{References}

Baldissera, F., Cavallari, P., \& Civaschi, P. (1983). Preferential coupling between voluntary movements of ipsilateral limbs. Neuroscience Letters, 34, 95-100.

Becker, W., \& Jurgens, B. (1979). An analysis of the saccadic system by means of double-step stimuli. Vision Research, 19, 967-983.

Benecke, R., Meyer, B. U., Gohmann, M., \& Conrad, B. (1988). Analysis of muscle responses elicited by transcranial stimulation of the cortico-spinal system in man. Electroencephalography and Clinical Neurophysiology, 69, 412-422.

Brooks, V. B. (1986). The neural basis of motor control. New York: Oxford University Press.

Bullock, D., \& Grossberg, S. (1988). Neural dynamics of planned arm movements: Emerging invariants and speed-accuracy properties during trajectory formation. Psychological Review, 95, $49-90$. 
Bullock, D., \& Grossberg, S. (1991). Adaptive neural networks for control of movement trajectories invariant under speed and force rescaling. Human Movement Science, 10, 3-53.

Chang, P., \& Hammond, G. R. (1987). Mutual interactions between speech and finger movements. Journal of Motor Behavior, 19, 265-274.

Cohen, J. D., Servan-Schreiber, D., \& McClelland, J. L. (1992). A parallel distributed processing approach to automaticity. The Americal Journal of Psychology, 105, 239-270.

Coles, M. G. H. (1989). Modern mind-brain reading: Psychophysiology, physiology, and cognition. Psychophysiology, 26, 251269.

De Jong, R. (1993). Multiple bottlenecks in overlapping task performance. Journal of Experimental Psychology: Human Perception and Performance, 19, 965-980.

De Jong, R., Coles, M. G. H., Logan, G. D., \& Gratton, G. (1990). In search of the point of no return: The control of response processes. Journal of Experimental Psychology: Human Perception and Performance, 16, 164-182.

De Jong, R., Wierda, M., Mulder, G., \& Mulder, L. J. M. (1988). Use of partial information in response processing. Journal of Experimental Psychology: Human Perception and Performance, 14, 682-692.

Georgopoulos, A. P., Kalaska, J. F., \& Massey, J. T. (1981). Spatial trajectories and reaction times of aimed movements: Effects of practice, uncertainty, and change in target location. Journal of Neurophysiology, 46, 725-743.

Gratton, G., Coles, M. G. H., \& Donchin, E. (1983). A new method for off-line removal of ocular artifact. Electroencephalography and Clinical Neurophysiology, 55, 468-484.

Gratton, G., Coles, M. G. H., Sirevaag, E., Eriksen, C. W., \& Donchin, E. (1988). Pre- and poststimulus activation of response channels: A psychophysiological analysis. Journal of Experimental Psychology: Human Perception and Performance, 14, 331-344.

Heuer, H. (1993). Structural constraints on bimanual movements. Psychological Research, 55, 83-98.

Jennings, J. R., van der Molen, M. W., Brock, K., \& Somsen, R. J. M. (1992). On the synchrony of stopping motor responses and delaying heartbeats. Journal of Experimental Psychology: $\mathrm{Hu}$ man Perception and Performance, 18, 422-436.

Kelso, J. A. S., Putnam, C. A., \& Goodman, D. (1983). On the space-time structure of human interlimb coordination. Quarterly Journal of Experimental Psychology, 35A, 347-375.

Kornblum, S., Hasbroucq, T., \& Osman, A. (1990). Dimensional overlap: Cognitive basis for stimulus-response compatibility-A model and taxonomy. Psychological Review, 97, 253270.

Logan, G. D. (1985). On the ability to inhibit simple thoughts and actions: II. Stop-signal studies of repetition priming. Journal of Experimental Psychology: Learning, Memory, and Cognition, 11, 675-691.

Logan, G. D. (1994). On the ability to inhibit thought and action: A users' guide to the stop signal paradigm, In D. Dagenbach \& T. H. Carr (Eds.), Inhibitory processes in attention, memory, and language (pp. 214-249). San Diego: Academic Press.
Logan, G. D., \& Burkell, J. (1986). Dependence and independence in responding to double stimulation: A comparison of stop, change, and dual-task paradigms. Journal of Experimental Psychology: Human Perception and Performance, 12, 549-563.

Logan, G. D., \& Cowan, W. B. (1984). On the ability to inhibit thought and action: A theory of an act of control. Psychological Review, 91, 295-327.

Logan, G. D., Kantowitz, B. H., \& Riegler, G. L. (1986). On the ability to stop selectively: Mechanisms of response interdiction in choice reaction time. Unpublished manuscript, Purdue University.

Meyer, D. E., Osman, A., Irwin, D. E., \& Yantis, S. (1988). Modern mental chronometry. Biological Psychology, 26, 3-67.

Newell, K. M. (1985). Coordination, control, and skill. In D. Goodman, R. B. Wilberg, \& I. M. Franks (Eds.), Differing perspectives in motor learning, memory, and control (pp. 295317). Amsterdam: North-Holland.

Osman, A., Kornblum, S., \& Meyer, D. E. (1986). The point of no return in choice reaction time: Controlled and ballistic stages of response preparation. Journal of Experimental Psychology: $\mathrm{Hu}$ man Perception and Performance, 12, 243-258.

Osman, A., \& Moore, C. (1993). The locus of dual-task interference: Psychological refractory effects on movement-related brain potentials. Joumal of Experimental Psychology: Human Perception and Performance, 19, 1292-1312.

Pashler, H., Carrier, M., \& Hoffman, J. (1993). Saccadic eye movements and dual-task interference. The Quarterly Journal of Experimental Psychology, 46A, 51-82.

Rohrbaugh, J. (1984). The orienting reflex: Performance and CNS manifestations. In R. Parasuraman and R. Davies (Eds.), Varieties of attention (pp. 323-373). New York: Academic Press.

Schiller, P. H., Sandell, J. H., \& Maunsell, J. H. R. (1987). The effect of frontal eye field and superior colliculus lesions on saccadic latencies in the rhesus monkey. Journal of Neurophysiology, 57, 1033-1049.

Smid, H. G. O. M. (1993). When action starts before perception is ready: A chronopsychophysiological approach. Unpublished doctoral thesis, University of Groningen, The Netherlands.

Soechting, J. F., \& Lacquaniti, E. (1983). Modification of trajectory of a pointing movement in response to a change in target location. Journal of Neurophysiology, 49, 548-564.

Swinnen, S. P. (1992). Coordination of upper-limb movement: A neuro-dynamics approach. In G. E. Stelmach \& J. Requin (Eds.), Tutorials in motor behavior II (pp. 695-711). Amsterdam: Elsevier.

van der Molen, M. W., Bashore, T. R., Halliday, R., \& Callaway, E. (1991). Chronopsychophysiology: Mental chronometry augmented with psychophysiological time markers. In J. R. Jennings \& M. G. H. Coles (Eds.), Handbook of cognitive psychophysiology: Central and autonomic nervous system approaches (pp. 9-178). Chichester, England: Wiley.

Received August 12, 1993

Revision received May 24, 1994 Accepted June 8, 1994 